\title{
LA INDUSTRIA AUDIOVISUAL ANTE EL DESPLIEGUE DEL 5G: EL CASO DE LA UNIÓN EUROPEA
}

\section{The Audiovisual Industry Facing 5 G Deployment: The Case of the European Union}

\author{
Dra. Carmina CRUSAFON \\ Universidad Autónoma de Barcelona, Profesora agregada, España \\ E-mail: carmina.crusafon@uab.cat \\ https://orcid.org/0000-0002-6348-0799
}

Fecha de recepción del artículo: 08/10/2018

Fecha de aceptación definitiva: 21/10/2018

\begin{abstract}
RESUMEN
La llegada de la tecnología 5G tendrá un impacto importante en la industria audiovisual, especialmente en el ámbito televisivo. El análisis del caso europeo pone de relieve la relevancia que tiene la gestión del espectro en las políticas digitales. Este artículo explica cómo se ha diseñado la política europea para el despliegue del 5G con la creación de un consorcio donde participan los principales actores del ecosistema digital. Asimismo, analiza los futuros escenarios a los que se deberá encontrar el sector audiovisual. Todo parece indicar que el año 2025 sería la fecha en la que se podría producir un cambio significativo, en especial en el escenario de la televisión digital terrestre.
\end{abstract}

Palabras clave: Unión Europea; tecnología 5G; audiovisual; espectro; dividendo digital.

\begin{abstract}
The arrival of $5 \mathrm{G}$ technology will have a significant impact on the audiovisual industry, especially in the television field. The analysis of the European case highlights the relevance of spectrum management in digital policies. This article explains how the European policy for the deployment of $5 \mathrm{G}$ has been de-signed with the creation of a consortium where the main actors of the digital ecosystem participate. It also analyzes the future scenarios which the audiovisual sector must meet. Everything seems to indicate that the year 2025 will be the date on which a significant change could take place, especially in the scenario of dig-ital terrestrial television.
\end{abstract}

Key words: European Union; 5G Technology; Audiovisual; Spectrum; Digital Dividend. 


\section{INTRODUCCIÓN}

La tecnología $5 \mathrm{G}$ es la red móvil de próxima generación que va a permitir crear innovación y generar un cambio significativo en todos los sectores económicos. Su despliegue se realiza a través de mejoras en la banda ancha móvil (eMBB), con un sistema de comunicaciones ultra-fiable y de baja latencia (URLLC), y una red de comunicaciones masivas entre máquinas (MMTC) ${ }^{1}$. Se espera que la tecnología $5 \mathrm{G}$ permita velocidades muy rápidas, conectividad masiva, y redes confiables y súper receptivas para los usuarios. Esto se podría aplicar en experiencias de realidad virtual (VR) y realidad aumentada (AR) a demanda, en vehículos sin conductor, en un seguimiento médico a distancia, en servicios avanzados de automatización industrial, entre otras muchas aplicaciones. El resultado sería la fase avanzada de lo que se conoce como la sociedad digital y una existencia real del Internet de las cosas.

En el centro de esta transformación tecnológica, la gestión del espectro se sitúa como el elemento central de las políticas digitales y le convierte en un recurso estratégico para cualquier país. De su buen desarrollo, dependerá el progreso de la economía digital. Para la industria audiovisual, la llegada del 5G también tendrá un impacto importante, no solo a nivel tecnológico, sino que afectará al conjunto de la cadena de valor. En especial, los cambios se empezarán a observar en el periodo del 2020 al 2030.

La Unión Europea ha puesto en marcha toda una estrategia para conseguir los máximos beneficios del 5G, vinculada al desarrollo completo del mercado único digital. El objetivo general se enmarca en la estrategia 'Europa 2020' para conseguir un crecimiento inteligente, sostenible e inclusivo (Comisión Europea, 2010). Su desarrollo afecta directamente a la industria audiovisual, en especial al sector televisivo. Vinculado a su despliegue, los dividendos digitales se convierten en elementos esenciales para garantizar el pleno desarrollo del 5G y la garantía para las emisiones televisivas digitales (Pujol, 2016).

Este artículo se plantea tres preguntas de investigación. La primera quiere explicar cómo se ha diseñado la estrategia de la Unión Europea para la tecnología 5G en el marco de la creación del Mercado Único Digital (MUD). La segunda quiere analizar la figura creada para implementar su desarrollo, un consorcio público privado en el que participan los principales actores en el ecosistema audiovisual. La tercera analiza los escenarios de futuro a los que deberá hacer frente la industria audiovisual con la implementación completa del 5G.

La perspectiva teórica se sitúa en el marco del estudio de las políticas de comunicación europeas. Se toma como referencia el análisis de las políticas de la Unión Europea (Donders, Pauwels y Loisen, 2014; Flew, Iosifidis y Stemeers, 2016). El enfoque se sitúa en el institucionalismo (Galperin, 2004, y Schneider y Aspinwall, 2001), que analiza el papel que desempeñan las instituciones europeas, en el desarrollo de la política; así como su impacto en la industria audiovisual (Bonet, 2016). El estudio se realiza desde la metodología del análisis de caso, con una revisión de los principales documentos de las instituciones europeas, de la industria audiovisual y de las informaciones periodísticas aparecidas en los medios internacionales de referencia. La estructura del artículo se divide en tres partes: la primera se dedica a la política europea del espectro; la segunda, al consorcio público privado del 5G, y la última se ocupa de los escenarios de futuro para la industria audiovisual. Las conclusiones dan respuesta a las cuestiones planteadas.

\footnotetext{
${ }^{1}$ Estas siglas en inglés responden a: eMBB (enhanced Mobile Broadband), URLLC (Ultra Reliable Low Latency Communications) y mMTC (massive Machine Type Communications).
}

(C) Ediciones Universidad de Salamanca / CC BY - NC ND $\quad$ Fonseca, Journal of Communication, n. 17, 2018, pp. 11-22 


\title{
2. LA POLÍTICA DEL ESPECTRO RADIOELÉCTRICO DE LA UNIÓN EUROPEA: EL DESPLIEGUE DEL 5G
}

La gestión del espectro radioeléctrico ha sido uno de los ejes centrales de la política europea de las comunicaciones. El espectro es un bien público y su gestión se ha considerado un tema estratégico para cualquier país. Además, el valor del espectro no es solo económico, sino también un habilitador social, porque «primero y, ante todo, es un facilitador crucial para la recepción e impartición de información e ideas» (Delaere y Cullell-March, 2014, p. 360).

En el caso de la Unión Europea, los inicios de la política sobre el espectro se sitúan en los años 1980 con el proceso de liberalización de las telecomunicaciones. La presentación del Libro Verde de la Comisión sobre la política del espectro de 1998 marcará el inicio de esta política comunitaria. Posteriormente dos fechas clave han determinado su evolución: la Directiva (2002/21/CE) relativa a un marco regulador sobre las comunicaciones electrónicas y su reforma del 2009 (2009/140/CE) que supone la consolidación de la política europea (Cullell-March, 2010).

Posteriormente, la gran demanda sobre el espectro que existe en la industria de telecomunicaciones y tecnológica coincide con un cambio en la presidencia de la Comisión Europea. En el 2014, el nuevo presidente Jean-Claude Juncker convierte a esta política en una de las prioridades de la Unión Europea. Sus declaraciones son una clara muestra: «Necesitamos una mayor coordinación en la gestión del espectro para que Europa pueda convertirse en un líder en el despliegue de redes 5G. Nuestra economía lo necesita, nuestras empresas lo necesitan para crecer y expandirse»².

Este interés se enmarca en la estrategia 'Europa 2020' con el propósito de conseguir consolidar el Mercado Único Digital.

\begin{abstract}
El principal motivo de estos esfuerzos es económico: el deseo de recuperar el liderazgo mundial en telecomunicaciones móviles, una posición que Europa mantuvo en la década de 1990 después de la adopción mundial del Sistema Global para Comunicaciones Móviles, originalmente denominado Groupe Spécial Mobile (GSM), el estándar de telefonía móvil de segunda generación $(2 \mathrm{G})$, pero que luego se perdió con la introducción de los sistemas 3G y 4G (Ala-Fossi y Bonet, 2018, pp. 337-338).
\end{abstract}

El desarrollo del mercado digital ha convertido a la banda UHF en el bien más preciado. En una primera etapa, el interés se centró en la banda $800 \mathrm{MHz}(790-862 \mathrm{MHz}$, o conocido como «el primer dividendo digital») resultado de la liberación de frecuencias con el apagón de la televisión analógica; posteriormente se focaliza en la banda $700 \mathrm{MHz}$. La Conferencia Mundial de Radiocomunicaciones de 2012 (CMR-12) decidió que esta banda debía atribuirse a los servicios móviles y de radiodifusión en la región 1 (Europa y África) a partir de 2015. En la actualidad, la banda de radiodifusión UHF comprende la gama de frecuencias de 470 a $790 \mathrm{MHz}$ que se utilizan para la televisión digital terrestre (TDT) y los equipos de audio para la creación de programas y eventos especiales (audio PMSE), esencialmente los micrófonos inalámbricos.

La importancia de la banda de transmisión en UHF en la Unión Europea viene justificada por dos motivos: el primero, porque la televisión sigue siendo el medio de comunicación dominante en el sistema mediático de países como España, Italia y Francia; y el segundo, por el papel trascendente de la radiodifusión televisiva en la política y la cultura europeas (Ala-Fossi y Bonet, 2018).

\footnotetext{
2 Estas declaraciones se encuentran en el folleto informativo sobre la política del espectro de la Comisión Europea (Fuente: https://ec.europa.eu/digital-single-market/en/news/spectrum-european-union).
}

(C) Ediciones Universidad de Salamanca / CC BY - NC ND $\quad$ Fonseca, Journal of Communication, n. 17, 2018, pp. 11-22 
A partir de la decisión CMR-12, la Comisión encargó un estudio sobre cuál debía ser la posición de la UE en este escenario. Este documento, conocido como «informe Lamy», recomendó reorientar la banda de $700 \mathrm{MHz}$ hacia la banda ancha inalámbrica, pero sin olvidar el apoyo al modelo audiovisual europeo. De esta manera se aseguraba que la TDT podría tener acceso a frecuencias inferiores a $700 \mathrm{MHz}$ hasta 2030, aunque se debería revisar el 2025. Además, el informe Lamy recomendaba la flexibilidad nacional en el uso del espectro, limitada solo al enlace descendente. Esto significa restringir todas las transmisiones en un sistema inalámbrico, con independencia de su tecnología, la comunicación unidireccional desde las estaciones de la infraestructura central, como una torre de radiodifusión de televisión o una estación base de telefonía móvil, terminales portátiles o móviles, tales como televisores o teléfonos móviles. El hecho debe estar sujeto a un régimen de autorización flexible que supone la posibilidad de que los titulares de derechos de uso del espectro comercialicen y alquilen sus derechos en el marco de la aplicación de los artículos 9, 9 bis y 9 ter de la Directiva 2002/21 /CE24. En resumen, se sugirió que incluso si la banda de $700 \mathrm{MHz}$ se abriera al uso móvil en toda Europa para 2020, se debería garantizar que las emisoras tengan prioridad para la banda UHF restante hasta 2030, con ciertas «opciones de flexibilidad» (Lamy, 2014, p.7-10).

Tabla 1: Fechas relevantes en la política de gestión del espectro de la UE para el despliegue 5G

\begin{tabular}{|c|c|}
\hline AÑ & ACCIÓN \\
\hline 2010 & Estrategia EUROPA 2020 para la creación de un mercado único digital \\
\hline 2014 & Informe Lamy \\
\hline 2015 & Primer dividendo digital (banda 800 Mhz) \\
\hline 2016 & Plan para el despliegue del 5G \\
\hline 2017 & Decisión del Consejo y del Parlamento Europeos para fijar liberar banda \\
& 700Mhz para el 5G \\
\hline 2018 & Adopción del nuevo código europeo de comunicaciones \\
\hline
\end{tabular}

Fuente: elaboración propia a partir de documentos de la Comisión Europea

La Comisión Europea (2016a y 2016b) presentó en septiembre de 2016 un Plan de Acción para $5 \mathrm{G}$ en Europa para demostrar que Europa tiene la ambición de liderar el despliegue de 5G y pasar a una sociedad de gigabits y asegurarse de que todos los europeos, ya sea en el campo o en las ciudades, puedan obtener acceso a una conexión a internet de calidad. Propuso que la banda de $700 \mathrm{MHz}$ se asignara a la banda ancha inalámbrica el 30 de junio 2020 como muy tarde en todos los países de la UE. Para cumplir este plazo, los Estados miembros debían adoptar y hacer públicos sus planes nacionales para la cobertura de la red y la conclusión de acuerdos de coordinación transfronteriza a finales de 2017. Estos planes tienen que preparar la transición y asegurar una buena cobertura de la red que ayudará a reducir la brecha digital y crear las condiciones necesarias para la cobertura de los vehículos conectados o cuidado de la salud a distancia.

Posteriormente, el Consejo Europeo (2017) y el Parlamento Europeo dieron luz verde a la propuesta entre marzo y mayo de 2017. A fecha de julio de 2018, tres Estados miembros (Francia, Alemania y Finlandia) ya habían autorizado el uso de la banda de $700 \mathrm{MHz}$ para servicios móviles. De los 25 Estados miembros de la UE restantes, 16 planean asignar la banda de $700 \mathrm{MHz}$ para junio de 2020, y otros 3 (Irlanda, Luxemburgo y España) estaban consultando sobre los términos (y el calendario) necesarios. Solo dos Estados miembros (Croacia y Letonia) han indicado que es poco probable que reasignen el espectro antes de junio de 2020 (Analysys Mason, 2018). 
El objetivo final es aprovechar las ventajas socioeconómicas de esta banda para reducir la brecha digital y contribuir a la generalización de la Internet de las Cosas. Para conseguir este mercado único digital, es esencial disponer de una conectividad inalámbrica de alta velocidad universal que pueda proporcionarse de la manera más eficiente a todos los ciudadanos de la UE a través de redes fijas e inalámbricas igualmente esenciales. La conectividad de alta velocidad en toda la UE permitirá desarrollar servicios innovadores en el mercado audiovisual y de las comunicaciones móviles.

Según los estudios encargados por la Comisión Europea, la próxima generación costaría de 1.200 a 4.400 millones de euros. La mayor parte de estos costes serían aportados por los usuarios finales cuando actualizaran sus equipos de recepción antes de que finalizara el ciclo de renovación normal de los mismos. Teniendo en cuenta los resultados de las últimas subastas del espectro de bandas de frecuencias de $800 \mathrm{MHz}$ y $900 \mathrm{MHz}$, una estimación conservadora de los ingresos globales procedentes de la asignación de la banda de frecuencias de $700 \mathrm{MHz}$ en 2020 se situaría en torno a los $11.000 \mathrm{mi}-$ llones de euros.

La UE tiene poderes para definir los aspectos técnicos del uso del espectro, pero los Estados miembros deciden cuándo se pondrá a disposición de los operadores móviles en su territorio y en qué condiciones, durante cuánto tiempo, a qué precio. En estas circunstancias, no es posible desplegar nuevos servicios de datos inalámbricos de alta calidad en toda Europa al mismo tiempo y con un modelo de negocio común que admita grandes inversiones. Se necesita una estrategia coordinada, fruto de las acciones entre los Estados miembros y las instituciones europeas. En el desarrollo de la política del espectro, se observa claramente la tendencia que apunta Michalis (2016) de un papel creciente del Estado regulador, bien ejemplificado en el caso de la Unión Europea, donde tanto la Comisión Europea como los Estados Miembros quieren ejercer un control férreo sobre la gestión del espectro, aunque no siempre con afanes armonizadores sino más bien liberalizadores del mercado.

\section{El MODELO PARA DEFINIR EL DESPLIEGUE DEL 5G EN LA UNIÓN EUROPEA: EL CONSORCIO PÚBLICO PRIVADO 5G PPP}

Una de las singularidades en el despliegue de la tecnología $5 \mathrm{G}$ es conocer cuál es la participación de diferentes actores. En el caso de la Unión Europea, se ha diseñado una estrategia conjunta específica entre la industria, la academia y las instituciones europeas. Se ha constituido el consorcio, el 5G Public Private Partnership (5G PPP) ${ }^{3}$, que se encarga de analizar las diferentes posibilidades y cómo implementarlas en territorio europeo. En concreto, la parte central de los ensayos y pilotos de $5 \mathrm{G}$ se realizará a través de ensayos privados (comerciales y precomercial) entre los operadores de red y los fabricantes, a la vez que participarán las diferentes industrias verticales que se ven afectadas por su despliegue (5G PPP, 2017). La elección de esta forma de actuación responde a las necesidades que impone la nueva gobernanza (Puppis, 2010) a la hora de plantear las políticas públicas y las actuaciones gubernamentales. Es necesaria una colaboración de todos los actores que participan en el ecosistema.

\footnotetext{
${ }^{3} \mathrm{La}$ iniciativa 5G PPP cuenta con la participación de 165 organizaciones y con un presupuesto de 700 millones de euros con el objetivo de posicionar a Europa como un mercado líder para el 5G. El 5G PPP se enmarca en el marco de investigación e innovación del programa Horizonte 2020 de la Unión Europea (UE). El Acuerdo Contractual 5G PPP fue firmado en diciembre de 2013 por la Comisión Europea, que representa el lado público, y la Asociación de Infraestructura 5G (5GIA), que representa el lado privado. El 5GIA se creó específicamente para firmar el contrato. Es una organización internacional sin ánimo de lucro con base en Bélgica y establecida bajo la ley belga. La membresía de 5GIA comprende 34 miembros electos, la mayoría de los cuales (17) son organizaciones de la industria, incluidos fabricantes y transportistas, algunas de las cuales son filiales europeas de compañías globales, como Huawei y NEC. También hay seis organismos de investigación, seis PYME y cinco «organizaciones adicionales», incluidas IBM, Intel y Samsung. El 5GIA también tiene un número de miembros asociados, que incluyen otras organizaciones de investigación, grupos de usuarios y organismos de estándares.
}

(C) Ediciones Universidad de Salamanca / CC BY - NC ND Fonseca, Journal of Communication, n. 17, 2018, pp. 11-22 
Esta acción se enmarca en la estrategia 'EU HORIZON 2020', la iniciativa de apoyo a la investigación y desarrollo, que tiene que ayudar a la consecución del mercado único digital. La primera acción que ha desarrollado el consorcio ha sido el 'Libro blanco sobre el 5G' para evaluar los sectores económicos verticales sobre los que podría tener más impacto. Se han seleccionado cinco ámbitos: las fábricas del futuro, la energía, el automóvil, la salud y el sector de los medios de comunicación y del entretenimiento (5G PPP, 2016). En relación con este último, su presentación se realizó en 2016 y señaló un conjunto de elementos como necesarios para un desarrollo completo de la tecnología 5G. Se integrará a las diferentes tecnologías de red (incluyendo el unicast, el multicast y broadcast) y ofrecerá todas las capacidades de almacenamiento en caché necesarias para proporcionar servicios de seguimiento y evaluación de todos los casos de uso.

El foco de la investigación en redes $5 \mathrm{G}$ hasta la fecha se ha dedicado en gran medida a los avances en arquitecturas de red, tecnologías e infraestructuras en todos los proyectos financiados en la Fase 1 de 5G-PPP. En la segunda fase, el esfuerzo se ha puesto en las aplicaciones y servicios que aprovechará y explotará las capacidades avanzadas del 5G y a partir de 2019 se empezarán a tener los resultados de los bancos de pruebas.

De las 21 iniciativas de la segunda fase del 5G PPP, dos tienen como eje central el despliegue de la nueva tecnología dentro del ámbito de los medios de comunicación, con especial impacto en el sector audiovisual. Estos son el 5G MEDIA y 5G - Xcast (véase tabla 2). Las ventajas de la perspectiva del ecosistema del $5 \mathrm{G}$ identifica los medios de comunicación como uno de los sectores centrales en el desarrollo tecnológico, no como un actor subsidiario, sino como uno de los sectores económicos centrales en la apuesta europea por $5 \mathrm{G}$.

Tabla 2: Los proyectos vinculados con el sector audiovisual del consocio 5G PPP

\begin{tabular}{|c|l|l|l|}
\hline \multicolumn{1}{|c|}{ Proyecto } & \multicolumn{1}{|c|}{ Concepto central } & \multicolumn{1}{|c|}{ Participantes } & \multicolumn{1}{c|}{$\begin{array}{c}\text { Empresa audiovisual } \\
\text { participante }\end{array}$} \\
\hline 5G MEDIA & Streaming como servicio & 15 instituciones & RTVE \\
\hline $5 G-$ Xcast & $\begin{array}{l}\text { Difusión y multidifusión } \\
\text { inalámbrica }\end{array}$ & 18 instituciones & BBC y UER \\
\hline
\end{tabular}

Fuente: Elaboración propia a partir de datos del EU 5G PPP

El objetivo de la iniciativa 5G MEDIA (5G PPP, 2017b) es introducir el concepto de streaming como servicio, que permita transmitir video de alta calidad en cualquier sitio, consumir contenido en cualquier momento y adaptar la calidad a cada dispositivo. De hecho, responde a la filosofía que se está imponiendo en el mundo digital que responde a las siglas en inglés 'AAA' (Anytime, Anywhere and Any Device). En el consorcio 5G MEDIA, participan 15 instituciones (empresas, universidades, medios de comunicación e institutos de investigación). Destacan una presencia de mayoritaria de empresas mediterráneas (Italia, Grecia y España) y solamente participa un único radiodifusor, Radio Televisión Española (RTVE).

En concreto, las aplicaciones que se prevén ofrecer con el 5G en el ámbito mediático y del entretenimiento se clasifican en las siguientes categorías: el uso de la Ultra-Alta fidelidad; los medios inmersivos e interactivos con adaptación inteligente al ambiente visual, que incluye la transmisión de vídeo en 3D; ofrecer contenido capturado y compartido inmediatamente; y diseñar experiencias completas 
multisensoriales de inmersión en el ámbito de los juegos (gaming), pasando de experiencia basada en casa a disfrutarla en cualquier lugar.

Asimismo, se están realizando tres estudios de uso que quieren tener impacto en diferentes partes de la cadena de valor de las empresas audiovisuales. El primero está centrado en ofrecer soluciones para una producción inteligente y remota a las televisiones que les permita utilizar herramientas para combinar la producción de contenidos profesionales con los creados por el usuario. Se han marcado dos escenarios: uno de producción en remoto y otro de contribución desde un móvil en las emisiones de video en directo (conexiones en vivo). El segundo se centra en crear aplicaciones inmersivas con el uso de la realidad virtual. En concreto, se trata de ofrecer experiencias participativas inmersivas. Esto es, en el entorno de los videojuegos, múltiples jugadores en distintas partes del planeta entran en un área 3D construida en tiempo real; y a su vez, experiencias de interacción inmersiva de los espectadores. Los jugadores pueden navegar dentro del mundo virtual del videojuego usando las posturas corporales. El tercer caso de uso ofrece nuevas capacidades a los proveedores de servicios mediáticos a través de la distribución de contenidos de ultra-alta calidad ( $4 \mathrm{~K}$ y $8 \mathrm{~K}$ ) con un consumo óptimo de los recursos. En este caso, el propósito es permitir pasar de un dispositivo de pantalla fija a uno móvil (My screens follows-me) y el segundo es permitir en una emisión televisiva en directo seleccionar los encuadres y los audios.

El proyecto 5G-XCast (5G PPP, 2018) está enfocado en los habilitadores de comunicaciones de difusión y multidifusión para la quinta generación de sistemas inalámbricos. En este proyecto participan 18 instituciones de 9 países, dos vinculadas con el sector audiovisual (BBC y la UER), cuatro operadores de telecomunicaciones, ocho empresas y 3 universidades. Su participación abarca el conjunto de la cadena de valor: la generación de contenidos (creación y producción), la distribución (funcionalidad central, proveedores de señal y de servicios) y al usuario final (a través de diferentes dispositivos). Además, también participan universidades y centros de investigación académicos.

El proyecto analiza primero los requisitos que deben tener los medios del futuro, incluidos los televisores de alta definición 4K/8K (UHDTV), HDR (rango dinámico alto), HFR (altas velocidades de cuadro) y de amplio espacio de color, de contenido basado en objetos, virtual, aumentado y de realidad mixta (VR / AR / MR), medios visuales de $360^{\circ}$ y audio de próxima generación, que abarcan aspectos comerciales y técnicos. Asimismo, definirá las especificaciones de nivel superior para el transporte de la señal, junto con las arquitecturas del sistema. Las interfaces comunes de programación de aplicaciones (API) se desarrollarán para permitir un acceso sin interrupciones a los contenidos y servicios en cualquier momento, en cualquier lugar y desde cualquier dispositivo.

Se han establecido tres bancos de pruebas. El primero, conocido bajo las siglas 5GIC, se basa en la radiodifusión basada en objetos y se llevará a cabo en el Reino Unido. Este banco de pruebas demostrará experimentalmente el uso del objeto basado difusión en colaboración con la filial de la BBC dedicada a los temas de investigación y desarrollo, la BBC R\&D. El segundo con las siglas IRT se basa en un servicio de radiodifusión híbrida, en colaboración con Nokia y BR, opera una red de frecuencia única eMBMS con cobertura urbana y rural en la ciudad y los alrededores de Múnich. El tercero se realiza desde Turku (Finlandia). La Universidad de Ciencias Aplicadas de Turku ha desarrollado un banco de pruebas para probar tecnologías y aplicaciones LTE /5G (5GTNT, 5G Test Network Tur$\mathrm{ku}$ ). Se realizan dos conjuntos de ensayos de transmisión de mensajes de alerta pública (alerta ámbar y alerta de advertencia pública multimedia).

Asimismo, ya se ha empezado a probar en eventos públicos como los Campeonatos Europa 2018 (de atletismo, deportes acuáticos, ciclismo, gimnasia, remo, triatlón, con un nuevo campeonato de equipos de golf) que se celebraron en agosto de 2018, y las pruebas fueron un éxito. Las previsiones 
son realizar pruebas en la feria IBC de noviembre de 2018 en Ámsterdam y en el Congreso Mundial de Móviles en febrero de 2019 en Barcelona.

\section{EL IMPACTO DEL 5G EN LA INDUSTRIA AUDIOVISUAL: LOS PRÓXIMOS ESCENARIOS}

El impacto de la tecnología $5 \mathrm{G}$ en la industria audiovisual no se podrá evaluar de forma completa hasta el año 2030. Mientras no se llega a este escenario, es necesario identificar otras fechas clave en los próximos años que pueden ir determinando los resultados concretos en función de los diferentes ámbitos de actuación.

A nivel internacional, el primer horizonte se sitúa en la Unión Internacional de Telecomunicaciones (UIT) donde el año próximo 2019 se celebrará la Conferencia Mundial de Radiocomunicaciones (CMR-19) con objeto de extender el servicio y los casos de uso del 5G a nuevas bandas. En un principio no se esperan cambios porque en la reunión anterior (CR-15) ya se determinó la importancia de esta banda para el sector audiovisual y se decidió que, aunque en la banda de $700 \mathrm{MHz}$ se eliminará de la radiodifusión para la utilización móvil también en la Región 1 de la UIT, no habrá cambios en el estado de la parte restante del espectro de la UHF (470-694 MHz) hasta que se haya revisado para la reunión (CMR- 23) que se debería celebrar en el 2023 (GSMA, 2017).

A nivel de la Unión Europea, en el 2019 se ha de producir la armonización técnica de las bandas de espectro 3.6 GHz (para corredores de automóviles conectados) y $26 \mathrm{GHz}$ (para banda ancha inalámbrica densa y mejorada). A mediados de 2020, se debe realizar la transposición del nuevo código de comunicaciones electrónicas a la legislación nacional, que incluye un límite de tiempo vinculante para hacer que el espectro esté disponible, revisión por pares, mayor duración de la licencia, etc.

En el 2020 la banda de $700 \mathrm{MHz}$ debe estar asignada en la mayoría de los Estados miembros, con al menos con el $5 \mathrm{G}$ en una ciudad por Estado miembro. Todos los Estados miembros asignarán la banda de $700 \mathrm{MHz}$ a mediados de 2020. Otras bandas $5 \mathrm{G}(3.6 \mathrm{GHz}$ y $26 \mathrm{GHz}$ ) deben asignarse antes del 31 de diciembre de 2020 .

El despliegue completo de la infraestructura 5G está planificado entre el 2020 y el 2025. De hecho, se habla del 2025 como la fecha para la consolidación de lo que se denomina como la «Gigabit Society», donde el $5 \mathrm{G}$ esté disponible en las principales ciudades y en las rutas de transporte más importantes.

A nivel europeo, el año 2020 se presenta como una fecha clave para enseñar al mundo los primeros resultados y se ha escogido un acontecimiento deportivo, UEFA EURO 2020, para mostrar las aplicaciones del 5G a las retransmisiones televisivas. Es decir, el futbol se convierte en el embajador y el banco de pruebas de esta tecnología. Además, normalmente este tipo de retransmisiones deportivas se incluyen en el listado de contenidos de interés general que establece la Directiva de Servicios de Comunicación Audiovisual. Esto es, el objetivo es que el EURO 2020 actúe como el «evento de lanzamiento» para 5G en la Unión Europea.

Los campeonatos de fútbol de la UEFA EURO 2020 se jugarán en 12 ciudades diferentes de Europa (Glasgow, Dublín, Copenhague, Budapest, Bucarest, Bilbao, Ámsterdam, San Petersburgo, Roma, Múnich, Bakú y Londres). Esto hace que el EURO 2020 sea una excelente oportunidad para una prueba paneuropea de 5G; también debido a la atención de los medios que recibirá. El calendario de EURO 2020 es verano de 2020, justo antes de la celebración de los Juegos Olímpicos de 2020 en Japón. En cada una de las ciudades, será necesario un consorcio con la participación de gobiernos locales, estadios, operadores, vendedores de infraestructura y proveedores de aplicaciones. Para cada 
ciudad que es sede del campeonato, se establece un acuerdo con (al menos un) operador para garantizar que habrá una cobertura $5 \mathrm{G}$ en la que se pueden probar los servicios $5 \mathrm{G}$ previstos.

Al mismo tiempo, los dos proyectos 5G MEDIA y 5G-Xcast también tienen previstos sus primeros resultados en el 2021 y la fase de despliegue completa y de comercialización se alargará hasta el 2024. A partir de esta fecha hasta el 2030 parece que el impacto más significativo se podría producir en el ámbito de las emisiones de TDT. Si nos atenemos a la fórmula flexible «20-25-30» del informe Lamy, el impacto estará determinado por los resultados de las pruebas piloto del consorcio 5G PPP en el ámbito de la UE; y a nivel global por los cambios que se puedan producir de la mano de los gigantes tecnológicos (Google, Apple, Facebook y Netflix) en el consumo multiplataforma, a través principalmente del teléfono móvil.

\section{CONCLUSIONES}

El futuro de la industria audiovisual pasa por ofrecer experiencias de carácter inmersivo e interactivo desde cualquier dispositivo en cualquier momento y lugar. Este desarrollo depende del despliegue completo de la tecnología 5G. Es decir, no es posible pensar en el avance de este sector sin tener en cuenta esta tecnología. De ahí la importancia no solamente de los aspectos tecnológicos y de su implantación, sino también de la puesta en marcha de acciones concretas para su despliegue en el marco de la política de gestión del espectro.

El caso de la Unión Europea es un buen ejemplo para mostrar los vínculos entre el desarrollo de las políticas de comunicación y el avance de una tecnología, en este caso el 5G. Además, hay que enfatizar en este escenario la importancia de salvaguardar espacio en el espectro para mantener las emisiones televisivas como parte del mandato de servicio público y garantizar su acceso al conjunto de la ciudadanía europea. Si bien está garantizado hasta el 2030, podría haber revisión en el 2023. Es importante señalar este aspecto que tiene que ver con los objetivos del bienestar político y social de las políticas públicas, así como en los elementos básicos para la garantía de una alta calidad democrática. En este sentido, el informe Lamy ya ponía de relieve esta necesidad y la decisión de las instituciones europeas ha sido de garantizarla. La clave estará en si esta determinación se seguirá manteniendo en los próximos años, porque tanto los Estados miembros quieren mantener un control férreo de las ondas como las empresas de telecomunicaciones presionan cada vez con más poder a las instituciones europeas para que pongan más espectro a su disposición.

El consorcio europeo creado 5G PPP es una muestra de la necesidad de abordar los nuevos desafíos tecnológicos desde una perspectiva de la nueva gobernanza. Esto es, a través de la participación de los diferentes actores (operadores de telecomunicaciones, radiodifusores, proveedores de servicios tecnológicos y de dispositivos), auspiciados por el apoyo de las instituciones europeas, principalmente a través del apoyo financiero. Esta ha sido la apuesta de la Unión Europea para conseguir un máximo desarrollo de esta tecnología. En los dos proyectos centrados en la parte audiovisual (5G MEDIA y 5G-Xcast), el enfoque es básicamente técnico con una preocupación central, de ofrecer una buena calidad de la experiencia, basada en conexiones sólidas, permanentes y de baja latencia, elemento fundamental en todo el planteamiento del despliegue del 5G. Estas dos iniciativas obligan a los medios a establecer estrategias colaborativas con operadores y empresas tecnológicas. Abre la perspectiva de los medios hacia otras áreas de aplicación que ahora mismo están desarrollando principalmente las empresas tecnológicas. Esta opción sitúa a los medios en el sector más puntero de la tecnología, pero requiere sobre todo de las empresas mediáticas más tradicionales un cambio de perspectiva que los haga mirar hacia un escenario de futuro muy cercano, sin olvidar la esencia de sus actividades. Tiene como 
factor de riesgo que la dimensión de las empresas y su capacidad financiera pueden convertirse en obstáculos para un pleno desarrollo.

En lo que se refiere a las dinámicas, el impacto no solamente vendrá determinado por los programas pilotos del 5G PPP; sino que habrá que tener en cuenta las decisiones que van tomando los gigantes tecnológicos, cuyas decisiones tienen realmente efectos globales. Asimismo, de la evolución del propio consumo audiovisual por parte de los usuarios/ciudadanos también dependerá el éxito del $5 \mathrm{G}$ y de la demanda que hagan de la TDT en el escenario europeo.

El caso europeo es una buena muestra de la nueva gobernanza con la participación de los distintos actores. La UE quiere recuperar el liderazgo con el 5G, aunque no lo tiene fácil por la competencia feroz que están ejerciendo EE. UU. y China con una apuesta decidida por esta tecnología y las inversiones realizadas en este ámbito. Veremos si la UE es capaz de competir y estar en el liderazgo global o sigue en segunda posición como pasó con el 3G y el 4G.

En definitiva, el futuro de la industria audiovisual pasa necesariamente por el despliegue del 5G para garantizar suficiente banda ancha inalámbrica para el consumo de contenidos de forma ubicua y constante. Asimismo, esta nueva tecnología determinará si las empresas audiovisuales tradicionales mantienen una posición relevante en la cadena de valor; o, por el contrario, su protagonismo se diluye en favor de los gigantes tecnológicos y/o las empresas de telecomunicaciones.

\section{BibLIOGRAFÍA}

Ala-Fossi, M. y Bonet, M. (2018). Who's Afraid of a Pan-European Spectrum Policy? The EU and the Battles Over the UHF Broadcast Band. International Journal of Communication, 12, 337-358.

Analysys Mason Consulting (2018). «The $700 \mathrm{MHz}$ band may largely be used for mobile in Europe by 2020 , but $5 \mathrm{G}$ everywhere by 2025 is unlikely», 25 de julio. Recuperado en:

http://www.analysysmason.com/About-Us/News/Newsletter/the-700mhz-band-by-2020$\mathrm{Jul18/}$

Bonet, M. (2016). El imperio del aire. Espectro radioeléctrico y radiodifusión. Barcelona: Editorial UOC.

Comisión Europea. (2016a). Propuesta de Decisión del Parlamento Europeo y del Consejo sobre el uso de la banda de frecuencias de 470-790 MHz en la Unio $\square$ n. SWD (2016) 19 final / SWD (2016) 20final. COM (2016) 43 FINAL, 2 febrero. Recuperado de: https://ec.europa.eu/transparency/regdoc/rep/1/2016/ES/1-2016-43-ES-F1-1.PDF

Comisión Europea. (2016b). Communication from the Commission to The European Parliament, The Council, The Eu- ropean Economic and Social Committee and The Committee of The Regions. 5G For Europe: An Action Plan. SWD/2016/0306 Final. Recuperado de http://eurlex.europa.eu/legal-content/EN/TXT/?uri=SWD:2016:306:FIN

Comisión Europea. (2010). Comunicación «Europa 2020: Una estrategia para un crecimiento inteligente, sostenible e integrador» [COM(2010) 2020 final de 3.3.2010]. Recuperado en: https://eurlex.europa.eu/legal-content/ES/TXT/?uri=LEGISSUM\%3Aem0028

Consejo europeo. (2017). «La UE coordina el espectro clave para impulsar la conectividad en la carrera hacia la $5 \mathrm{G} »$, nota de prensa, 25 de abril. Recuperado de:

http://www.consilium.europa.eu/es/press/press-releases/2017/04/25-mobile-connec- tivity$5 \mathrm{~g}$-technology/

Cullell-March, C. (2010). La politica del espectro radioeléctrico en la Unión Europea: la armonización del dividendo digital en el Reino Unido y España. Tesis doctoral. Barcelona: Universitat Internacional de Catalunya. 
Delaere, S. y Cullell-March, C. (2014). Radio Spectrum Policy in the EU: Concepts, Trends, Issues, en Donders, K, Pauwels, C. y Loisen, J., The Palgrave Handbook of European Media Policy (pp. 360-381). London: Palgrave Macmillan.

Donders, K, Pauwels, C. y Loisen, J. (eds.). (2014). The Palgrave Handbook of European Media Policy. London: Palgrave Macmillan.

Digital TV Europe. (2016). «EC and industry groups set out 5G vision», July 8, Recuperado de https://www.digitaltveurope.com/2016/07/08/ec-and-industry-groups-set-out-5g-vision/

European Broadcasting Union. (2018). Trials Tests and Projects Relating To 4G/5G Broadcast Supported by European PSB Technical Report, Julio. Ginebra: EBU. Recuperado de https://tech.ebu.ch/docs/techreports/tr044.pdf

European Commission - Radio Spectrum Policy Group (2016). «Strategy Roadmap Towards 5G for Europe - Opinion on spectrum related aspects for next-generation wireless systems (5G)», RSPG16-032 FINAL, November 2016. URL: http://rspg-spectrum.eu/wpcontent/uploads/2013/05/RPSG16-032-Opinion 5G.pdf

Flew, T., Iosifidis P. y Steemers, J. (eds.). (2016). Global Media and National Policies. The return of the State. London: Macmillan Palgrave

Galperin, H. (2004). Beyond interests, ideas, and technology: An institutional approach to communication and information policy. The Information Society: An International Journal, 20(3), 159-168.

GSMA. (2017). La serie CMR Posicionamiento de Política Pública de GSMA. Conferencia Mundial de Radiocomunicaciones 2019. Visión de la industria móvil sobre el punto de agenda 1.13 Resumen ejecutivo. Recuperado de https://www.gsma.com/spectrum/wp-content/uploads/2017/09/AI1.13-Policy-Position-SPA.pdf

Lamy, P. (2014). Results of the work of the High Level Group on the future use of the UHF band (470-790 MHz). Report to the European Commission, Brussels, Belgium. Recuperado de: http://ec.europa.eu/information society/newsroom/cf/dae/document.cfm?doc id=6721

5G Infrastructure Association (5G-IA). (2018). 5G Pan-European Trials Roadmap Version 3.0, 5 de mayo. Recuperado de: https://5g-ppp.eu/wp-content/uploads/2018/05/5GInfraPPP TrialsWG Roadmap Version3.0.pdf

Michalis, M. (2016). Global Communications and National Policies: The View from the EU. En Flew, T., Iosifidis P. y Steemers, J. (eds.), Global Media and National Policies. The return of the State (pp. 122-137). London: Macmillan Palgrave.

Pujol, J. (2016). Espectro y frecuencias. Breve guía para no iniciados. En Bonet, M., El imperio del aire. Espectro radioeléctrico y radiodifusión. Barcelona: Editorial UOC.

Puppis, M. (2010). Media Governance: A New Concept for the Analysis of Media Policy and Regulation. Communication, Culture and Critique, 3(2), 134-149.

Schneider, G. y Aspinwal, M. (eds.). (2001). The Rules of Integration: The Institutionalist Approach to European Studies. Manchester: Manchester University Press.

5G Private Public Partnership (5G PPP). (2018). 5G-Xcast project details, folleto informativo. Recuperado de: http://5g-xcast.eu/wp-content/uploads/2018/09/5G Xcast Brochure Designer version.pdf

5G Private Public Partnership (5G PPP). (2017a). 5G Innovations for new Business Opportunities, marzo. Recuperado en https://5g-ppp.eu/roadmaps

5G Private Public Partnership (5G PPP). (2017b). Media Industry Meets 5G, folleto informativo. Recuperado en http://www.5gmedia.eu/cms/wp-content/uploads/2017/12/Media-industrymeets-5G-the-5G-MEDIA-project-in-5G-PPP-phase-2.pdf 
5G Private Public Partnership (5G PPP). (2016) 5G-PPP White Paper on Media \& Entertainment Vertical Sector, enero. Recuperado en https://5g-ppp.eu/wp-content/uploads/2016/02/5GPPP-White-Paper-on-Media-Entertainment-Vertical-Sector.pdf 